\title{
The Roles of Ultrasound-Based Radiomics In Precision Diagnosis and Treatment of Different Cancers: A Literature Review
}

\author{
Bing Mao, MD ${ }^{a, b}$, Shaobo Duan, MD ${ }^{b, c}$, Ruiqing Liu, MD ${ }^{b, d}, \mathrm{Na} L i$, PhD $^{b}$, Yaqiong Li, PhD ${ }^{b, e}$, Lianzhong Zhang, MD ${ }^{b, c, *}$ \\ ${ }^{a}$ Department of Public Development, Henan Provincial People's Hospital; Zhengzhou University People's Hospital; Henan University \\ People's Hospital, Zhengzhou, Henan, China $;{ }^{b}$ Henan Engineering Technology Research Center of Ultrasonic Molecular Imaging and \\ Nanotechnology, Zhengzhou, Henan China; ${ }^{c}$ Department of Ultrasound, Henan Provincial People's Hospital; Zhengzhou University \\ People's Hospital; Henan University People's Hospital, Zhengzhou, Henan, China; ${ }^{d}$ Department of Interventional Therapy, Henan \\ Provincial People's Hospital; Zhengzhou University People's Hospital; Henan University People's Hospital, Zhengzhou, Henan, China; \\ 'Department of Pharmacy, Henan Provincial People's Hospital; Zhengzhou University People's Hospital; Henan University People's
} Hospital, Zhengzhou, Henan, China

Received June 5, 2020; revision received June 26; accepted August 31, 2020

\begin{abstract}
The study aims to review literatures on ultrasound-based radiomics, including ultrasound modalities, and discusses basic methods, applications, and limitations of ultrasound-based radiomics. The search strategy was conducted in form of "Radiomics [Title/Abstract] and Ultrasound [Title/Abstract]"in PubMed. The retrieved articles were initially screened via abstracts. Then, the main objectives, methods, and achievements of selected articles were summarized. Finally, twenty articles focused on malignancies of different organs, such as liver, rectum, breast, and thyroid were included into this review. The multiparametric features exhibited a superior diagnostic performance compared with a single modality. Ultrasound-based radiomics can assist radiologists to improve the accuracy of diagnosis, and it may promote the development of precision diagnosis and treatment of various types of cancer.
\end{abstract}

Keywords: Ultrasound-based radiomics; Precision medicine; Data mining; Multi-parametric features

Advanced Ultrasound in Diagnosis and Therapy 2020;04:291-296

DOI: 10.37015/AUDT.2020.200051

\section{Introduction}

Non-communicable diseases, namely chronic diseases, account for the majority of global deaths, and as a main category of noncommunicable diseases, cancer, is now one of the leading causes of death and the most important barrier to increase life expectancy in the 21 st century. According to the statistics presented by the World Health Organization (WHO) in 2015, cancer ranks the first or second leading cause of death before age 70 years among 91 countries [1].

Using genomics and proteomics techniques to describe molecular characteristics is a hot topic in personalized diagnosis and treatment of cancer. However, due to the spatiotemporal heterogeneity of tumors, genomics and proteomics have limitations. They both require the analysis of a small portion of tumor tissues by invasive tissue puncture or surgery, and those small samples cannot capture the complete characteristics of the tumor. Medical imaging, in contrast to genomics and proteomics, assesses the characteristics of human tissues in a non-invasive manner and is often used in tumor diagnosis and treatment in clinical practice [2]. Medical imaging is one of the main factors that promote medical development and treatment [3]. The key goal

* Corresponding author: Department of Ultrasound, Henan Provincial People's Hospital, Zhengzhou University People's Hospital, Henan University People's Hospital, No. 7, Weiwu Rd., Jinshui Dist., Zhengzhou, Henan, 450003, China.

e-mail: zlz8777@163.com unrestricted use, distribution and reproduction in any medium provided that the original work is properly attributed. 
of medical imaging is "precision medicine", in which treatment is tailored to specific characteristics of patients and their disease [4]. Medical imaging can provide more comprehensive information about the whole tumor, and it can continuously monitor the development trend and treatment response of the disease. Therefore, medical imaging has great potential to guide the diagnosis and treatment of tumors.

In clinical practice, subjective terms such as "massive necrosis", "irregular margin", and "enlarged lymph node" are often used to describe the tumor tissue. In recent years, with the development of image acquisition, standardization, and statistical analysis, it is feasible to make quantitative analysis objectively and accurately.

Radiomics refers to the process of extraction and analysis of large amounts of advanced quantitative imaging features. It allows high-throughput extraction of quantitative features used to convert images into mineable data [5-7]. Radiomics has been justified to provide new perspectives on precision medicine in oncologic practice related to disease-free survival (DFS) [8-10], metastasis prediction [11-13], and therapeutic response assessment [14-16].

Although radiomics has shown promising results, it still faces limitations and challenges, such as heterogeneous study population, limited study samples, and lack of verified samples. Thus, for promoting the application of radiomics to the routine clinical practice, further researches need to be conducted to overcome the existing limitations and challenges.

Ultrasound possesses the advantages of no radiation, easy to carry out, which has become an integral part of medical practice [17], while a limited number of scholars have concentrated on ultrasound-based radiomics. This review aimed to analyze literature on ultrasoundbased radiomics, including ultrasound modalities, and discuss basic methods, applications, and limitations of ultrasound-based radiomics.

\section{Definition of Radiomics}

Radiomics is the process of extraction and analysis of large amounts of advanced quantitative imaging features with high throughput from medical images, such as computed tomography (CT), positron emission tomography (PET), and magnetic resonance imaging (MRI). The hypothesis is that quantitative analysis of medical imaging can provide more information than the qualitative description using naked eyes [18]. The radiomics process contains imaging acquisition, ROI segmentation, feature extraction, selection, and model establishment.

\section{Methods and Applications of Ultrasound-}

\section{Based Radiomics}

\section{Ultrasound-based radiomics for liver cancer}

Six studies have reported ultrasound-based radiomics models for liver cancer, which focused on the prediction of microvascular invasion [17,19], assessment of hepatic fibrosis[20,21], evaluation of biological characteristics of intrahepatic cholangiocarcinoma [22], and preoperative prediction of hepatocellular carcinoma (HCC) [23].

$\mathrm{HCC}$ is one of the most common hepatic tumors, accounting for nearly $90 \%$ of primary liver cancer cases, and it is the second leading cause of cancerrelated death worldwide [24]. Recurrence occurs in almost half of the patients within 2 years after surgery, and it has become the major cause of mortality [25]. Microvascular invasion (MVI) is defined as the presence of micrometastatic emboli within the vessels of the liver [26]. MVI was proved to be an important factor for predicting early recurrence and evaluating overall survival [27]. Preoperative prediction of MVI based on radiomics has markedly attracted scholars' attention. $\mathrm{Hu}$ et al. developed an ultrasound-based radiomics score for preoperative prediction of MVI in $\mathrm{HCC}$, and it was found that radiomics scores, alpha-fetoprotein (AFP), and tumor size were independent biomarkers of MVI $[17,19]$. The radiomics nomogram based on these three factors showed superior performance with area under the curve (AUC) value of 0.731 than the clinical nomogram (AUC, 0.634). Their findings concluded that the radiomics score combined with clinical factors could predict the MVI. Dong et al. also conducted a study aiming to predict the MVI in HCC lesions. The major difference between these two researches is that Dong et al. calculated three ultrasound feature maps of the original radio frequency (ORF) signals using signal analysis and processing (SAP) [19]. Their results revealed that imaging performance based on ORF was beyond that based on grayscale images.

Liver fibrosis and cirrhosis are growing health problems with rising mortality worldwide. Li et al. used multi-parametric ultrasound data including conventional radiomics, original radiofrequency, and contrastenhanced micro-flow (CEMF) to evaluate liver fibrosis [21]. Pairwise correlation and hierarchical clustering were undertaken to select features. The performance of ultrasound-based radiomics models combined with the selected features was evaluated by AUC. Their outcomes showed that ORF and CEMF features had superior performance than conventional radiomics for staging liver fibrosis. Besides, CEMF and ORF exhibited the greatest diagnostic value for the activity stage 
and steatosis stage, respectively. The model of multiparametric features unveiled a better performance than a single modality in classifying fibrosis. D'Souza et al. quantitatively analyzed computer-extracted features of B-mode ultrasound (BMUS) as a non-invasive tool to characterize hepatic fibrosis [20]. They extracted quantitative features, including heterogeneity, anisotropy, hepatorenal index, and echo intensity. Their results uncovered that BMUS imaging can be used in clinical settings as an accurate non-invasive method for fibrosis assessment.

Yao et al. established a radiomics-based analysis system for the diagnosis and clinical behavior prediction of HCC based on multi-parametric ultrasound imaging, including BMUS, shear wave elastography (SWE), and shear wave viscosity (SWV) imaging [23]. Besides, 2,560 features were extracted from the multi-modal ultrasound images for each patient. They used sparse representation theory (SRT) and support vector machine (SVM) to establish a prediction model for classification of benign and malignant focal liver lesions (FLLs), pathologic diagnosis of HCC, and clinical prognostic prediction. Their results showed that the performance of each model was improved when the viscosity was included and multi-modal ultrasound images could be used to evaluate clinical prognosis and differential diagnosis.

Peng et al. used ultrasound-based radiomics to assess the biological behaviors of intrahepatic cholangiocarcinoma (ICC) in a noninvasive manner [22]. It was demonstrated that radiomics signatures with moderate efficiency are helpful for predicting the biological behaviors of ICC noninvasively.

\section{Ultrasound-based radiomics for breast cancer}

Breast cancer is one of the most frequent cancer among women, with the greatest number of cancerrelated deaths [28]. Five studies have reported ultrasound-based radiomics models related to breast cancer. Those studies mainly focused on the prediction of biological behavior in invasive ductal carcinoma (IDC) [29] , the differential diagnosis between triple-negative breast cancer and fibroadenoma [30], differentiation of benign and malignant breast tumors [31,32], and the diagnosis of axillary lymph node metastasis in earlystage IDC.

IDC is one of the most common types of breast cancer, accounting for almost $80 \%$ of breast cancer [33]. Guo et al. evaluated the relationship between ultrasound features and biological behavior in 215 patients with IDC using the radiomics approach [29]. The tumors were automatically segmented by a phase-based active contour model. The high-throughput radiomics features were designed and extracted based on Breast Imaging-
Reporting and Data System (BI-RADS), and further selected according to t-test, inter-feature coefficients, and a least absolute shrinkage and selection operator (LASSO) regression model. The SVM classifier with three-fold-cross-validation was used to evaluate the relationship. The results showed that hormone receptor positive and human epidermal growth factor-2 (HER-2)negative tumors have different ultrasound features from triple-negative breast cancer (TNBC).

TNBC does not express the estrogen receptor (ER) and progesterone receptor (PR) or overexpress HER2 [34]. TNBC accounts for $20 \%$ of all the breast cancer cases and has the highest rate of recurrence and the worst prognosis [35]. Lee et al. distinguished TNBC from fibroadenoma using ultrasound texture features [30]. Their cohort study population included 715 fibroadenoma and 186 TNBC patients who were confirmed by pathology. A total of 730 radiomics features, including 14 intensity-based features, 132 textural features, and 584 wavelet-based features, were extracted. They developed the radiomics score by using penalized logistic regression with a LASSO model from those 730 extracted features. The findings unveiled that a radiomics score based on ultrasound texture analysis presented a high diagnostic performance in the differential diagnosis of fibroadenoma from TNBC.

Li et al. developed a radiomics approach, leveraging multimodal ultrasound images to improve the classification accuracy of breast tumors. B-mode ultrasound, SWE, and contrast-enhanced ultrasound images of 178 patients with 181 tumors (67 malignant and 114 benign) were included [32]. A total of 1,226 radiomics features were extracted and analyzed with attribute bagging. The results indicated that radiomics with attribute bagging combined with multimodal ultrasound images has the potential to be used for accurate diagnosis of breast tumors.

Luo et al. developed a nomogram combined with ultrasound-based radiomics and the BI-RADS for distinguishing benign from malignant breast tumors [31]. A total of 315 patients confirmed by postoperative pathology were included in the cohort study. 1,044 radiomics features were extracted from each ROI. Additionally, LASSO method was used to select features, and the radiomics score was calculated for each patient using 9 potential features. Their results showed that the nomogram combining the radiomics score and the BIRADS category was potentially helpful for predicting breast malignancy.

$\mathrm{Yu}$ et al. established a radiomics nomogram integrating clinical factors and radiomics features from ultrasound for the preoperative diagnosis axillary lymph node status in patients with early-stage invasive breast cancer 
(EIBC) [36] . A total of 426 EIBC patients confirmed by postoperative pathology were included in the cohort study. Besides, 96 radiomics features were extracted from each ultrasound image, and 14 features were selected using a LASSO logistic regression model. Their outcomes showed that ultrasound-based radiomics could predict axillary lymph node metastasis in breast cancer.

\section{Ultrasound-based radiomics for rectal cancer}

In recent years, rectal cancer has ranked among the top five common types of cancer, and it is the fifth leading cause of cancer-related deaths universally [37]. Three studies have reported radiomics models related to rectal cancer. Those researches focused on the prediction of tumor deposits [38], lymph node metastasis [39], and the diagnosis of gastrointestinal stromal tumors (GISTs) [40].

Tumor deposits are defined as focal aggregates of adenocarcinoma located in the pericolic or perirectal fat discontinuous with the primary tumor and unassociated with a lymph node [41]. Chen et al. predicted tumor deposits using a machine learning-based ultrasound radiomics model [38]. One hundred and twenty seven patients with rectal cancer were prospectively enrolled, and endorectal ultrasound and SWE data were collected for each patient. A total of 4,176 features were extracted, and LASSO method was used to reduce features, and then, a single-layer artificial neural network was constructed. Their outcomes revealed that ultrasound radiomics could accurately predict tumor deposits before therapy.

Predicting the nodal status of rectal tumors has remained challenging [42]. Chen et al. established a multi-parametric radiomics of the rectal tumor for the preoperative prediction of lymph node metastasis[39]. Moreover, 115 consecutive patients with rectal cancer were enrolled, and endorectal ultrasound, CT, and SWE data were collected for each patient. LASSO method was applied to reduce the dimension of the radiomics features, and the number of risk predictors of endorectal ultrasound, CT, and SWE was 3, 6, and 7, respectively. The risk predictors were verified by multivariate logistic regression combined with clinical data. The findings demonstrated that all three scores were higher in patients with lymph node metastasis than those in patients with negative lymph metastasis, and multi-parametric radiomics could predict lymph metastasis preoperatively, with better accuracy compared with conventional radiomics.

GISTs account for only approximately $1-3 \%$ of all gastrointestinal tumors, although rare, they are the most common mesenchymal tumors of the gastrointestinal tract [43]. Li et al. proposed a radiomics method to differentiate GISTs of the higher-risk group from the lower-risk group on endoscopic ultrasound images[40]. For this purpose, 168 higher-risk patients and 747 lowerrisk patients with GISTs were prospectively enrolled from 19 hospitals. A total of 439 radiomics features were extracted from endoscopic ultrasound images of each patient. LASSO method with 10 -fold cross-validation was used to reduce the dimension of the radiomics features, and the random forest algorithm was applied to establish the prediction model. The outcomes showed that the radiomics model could enhance preoperative diagnostic accuracy.

\section{Ultrasound-based radiomics for thyroid cancer}

Thyroid cancer has ranked ninth in incidence, and its etiology has not been well understood [37]. In China, the incidence of thyroid carcinoma has been reported as 9.0 per 10,000 , with a mortality rate of 6.8 per 100,000 [44]. Four studies have assessed radiomics models related to thyroid cancer. These studies focused on prediction of malignancy [45], lymph node metastasis [46,47], and DFS [48].

Sonographic features, such as micro-calcification, solid composition, taller shape, and irregular margin are considered as typical features for papillary thyroid carcinoma (PTC)[49,50]. Liang et al. developed an ultrasound-based radiomics score to predict the probability for malignancy of thyroid nodules compared with Thyroid Imaging, Reporting and Data System (TIRADS) scoring criteria [45]. Two hundred and thirty-two patients with pathologically proven thyroid nodules were prospectively enrolled from two hospitals. A total of 1,044 radiomics features were extracted from ultrasound images of each patient. LASSO method was used to reduce the dimension of the radiomics features, and 19 features were eventually detected as potential predictors. The univariate logistic regression analysis was applied to establish a prediction model based on the radiomics score and the TI-RADS score. The results showed that the radiomics score outperformed in predicting malignancy of thyroid nodules than TI-RADS.

PTC is the most common malignant neoplasm of the thyroid, accounting for the majority of patients diagnosed with thyroid cancer [51]. Park et al. developed an ultrasound-based radiomics signature to estimate DFS in patients with PTC[48]. They recruited 768 patients with PTC. A total of 730 radiomics features were extracted. LASSO method with 10 -fold cross-validation was used to reduce the dimension of the radiomics features, and 40 features were used to calculate the radiomics score. Their outcomes showed that ultrasound-based radiomics could be used for risk stratification in patients with PTC.

Lymph node metastasis is the most related risk factor for recurrence, and assessment of lymph node metastasis 
is of great clinical significance. However, there were no discriminative ultrasound features to estimate the status of lymph node metastasis[52]. Liu et al. developed the ultrasound-based radiomics to evaluate the status of lymph node metastasis [46,47]. In 2018, they investigated whether the application of multi-modality images including BMUS and strain elastography can improve the discriminability of thyroid tumors with lymph node metastasis based on a radiomics approach. A total of 684 ultrasound-based radiomics features were extracted, and a sparse representation coefficient-based feature selection method with 10-bootstrap was used to reduce the dimension of radiomics features. The SVM was applied to construct the prediction model. The results showed that multi-modality images can improve the accuracy required for estimation of the status of lymph node metastasis for PTC patients. In 2019, they collected ultrasound images of 445 patients with PTC to predict the status of lymph node metastasis. Six hundred and fourteen ultrasound-based features were extracted, and SVM classifier was employed to build and validate the prediction model. Their findings showed that ultrasoundbased radiomics could noninvasively predict the status of lymph node metastasis for PTC patients.

\section{Future Directions}

Radiomics refers to the extraction of high-throughput, quantitative features from clinical images, radiogenomics can explore the underlying relations between radiomic features at the tissue scale and molecular features at the genomic, transcriptomic, or proteomic level. In the future, radiogenomics could be the future direction and it will discover useful biomarkers to improve clinical decision-making and precision medicine.

\section{Conclusions}

Ultrasound-based radiomics has accomplished great advancements in the precision diagnosis and treatment of cancer, which mainly focused on malignancies of various organs, including liver, breast, rectal, and thyroid. The multi-parametric features exhibited a superior diagnostic performance than a single modality. The radiomics will positively assist radiologists to improve the accuracy of diagnosis.

\section{Acknowledgements}

This work was supported by the national key research and development program of China (Grant No. 2018YFC0114606) and the National Natural Science Foundation of China (Grant NO. 71974065). We wish to appreciate Dr. Yang Hai from Thomas Jefferson University for revising and editing our manuscript.

\section{Conflict of Interest}

The authors declare that there is no conflict of interest.

\section{Reference}

[1] Siegel RL, Miller KD, Jemal A. Cancer statistics, 2018. CA Cancer J Clin 2018; 68: 7-30.

[2] Kurland BF, Gerstner ER, Mountz JM, Schwartz LH, Ryan CW, Graham MM, et al. Promise and pitfalls of quantitative imaging in oncology clinical trials. Magn Reson Imaging 2012; 30: 1301-1312.

[3] Aerts HJ, Velazquez ER, Leijenaar RT, Parmar C, Grossmann P, Carvalho $\mathrm{S}$, et al. Decoding tumour phenotype by noninvasive imaging using a quantitative radiomics approach. Nat Commun 2014; 5: 4006.

[4] Lambin P, van Stiphout RG, Starmans MH, Rios-Velazquez E, Nalbantov G, Aerts HJ, et al. Predicting outcomes in radiation oncology--multifactorial decision support systems. Nat Rev Clin Oncol 2013; 10: 27-40

[5] Gillies RJ, Kinahan PE, Hricak H. Radiomics: Images Are More than Pictures, They Are Data. Radiology 2016; 278: 563-577.

[6] Lambin P, Leijenaar RTH, Deist TM, Peerlings J, de Jong EEC, van Timmeren J, et al. Radiomics: the bridge between medical imaging and personalized medicine. Nat Rev Clin Oncol 2017; 14: 749-762.

[7] Kumar V, Gu Y, Basu S, Berglund A, Eschrich SA, Schabath MB, et al. Radiomics: the process and the challenges. Magn Reson Imaging 2012; 30: 1234-1248.

[8] Huang Y, Liu Z, He L, Chen X, Pan D, Ma Z, et al. Radiomics signature: a potential biomarker for the prediction of disease-free survival in early-stage (I or II) non-small cell lung cancer. Radiology 2016; 281: 947-957.

[9] Akai H, Yasaka K, Kunimatsu A, Nojima M, Kokudo T, Kokudo $\mathrm{N}$, et al. Predicting prognosis of resected hepatocellular carcinoma by radiomics analysis with random survival forest. Diagn Interv Imaging 2018; 99: 643-651.

[10] Jiang Y, Chen C, Xie J, Wang W, Zha X, Lv W, et al. Radiomics signature of computed tomography imaging for prediction of survival and chemotherapeutic benefits in gastric cancer. EBioMedicine 2018; 36: $171-182$.

[11] Laghi A, Voena C. CT-based radiomics for biliary tract cancer: a possible solution for predicting lymph node metastases. Radiology 2019; 290: 99-100.

[12] Ortiz-Ramón R, Larroza A, Ruiz-España S, Arana E, Moratal D Classifying brain metastases by their primary site of origin using a radiomics approach based on texture analysis: a feasibility study. Eur Radiol 2018; 28: 4514-4523.

[13] Wang Y, Yu B, Zhong F, Guo Q, Li K, Hou Y, et al. MRI-based texture analysis of the primary tumor for pre-treatment prediction of bone metastases in prostate cancer. Magn Reson Imaging 2019; 60: 76-84.

[14] Jin X, Zheng X, Chen D, Jin J, Zhu G, Deng X, et al. Prediction of response after chemoradiation for esophageal cancer using a combination of dosimetry and CT radiomics. Eur Radiol 2019; 29: 6080-6088

[15] Horvat N, Veeraraghavan H, Khan M, Blazic I, Zheng J, Capanu $\mathrm{M}$, et al. MR imaging of rectal cancer: radiomics analysis to assess treatment response after neoadjuvant therapy. Radiology 2018; 287: 833-843.

[16] Fan Y, Jiang S, Hua M, Feng S, Feng M, Wang R. Machine learningbased radiomics predicts radiotherapeutic response in patients with acromegaly. Front Endocrinol (Lausanne) 2019; 10: 588.

[17] Hu HT, Wang Z, Huang XW, Chen SL, Zheng X, Ruan SM, et al. Ultrasound-based radiomics score: a potential biomarker for the 
prediction of microvascular invasion in hepatocellular carcinoma. Eur Radiol 2019; 29: 2890-2901.

[18] Lambin P, Rios-Velazquez E, Leijenaar R, Carvalho S, van Stiphout RG, Granton P, et al. Radiomics: extracting more information from medical images using advanced feature analysis. Eur J Cancer 2012; 48: 441-446.

[19] Dong Y, Wang QM, Li Q, Li LY, Zhang Q, Yao Z, et al. Preoperative prediction of microvascular invasion of hepatocellular carcinoma: radiomics algorithm based on ultrasound original radio frequency signals. Front Oncol 2019; 9: 1203.

[20] D'Souza JC, Sultan LR, Hunt SJ, Schultz SM, Brice AK, Wood $\mathrm{AKW}$, et al. B-mode ultrasound for the assessment of hepatic fibrosis: a quantitative multiparametric analysis for a radiomics approach. $S c i$ Rep 2019; 9: 8708.

[21] Li W, Huang Y, Zhuang BW, Liu GJ, Hu HT, Li X, et al. Multiparametric ultrasomics of significant liver fibrosis: A machine learning-based analysis. Eur Radiol 2019; 29: 1496-1506.

[22] Peng YT, Zhou CY, Lin P, Wen DY, Wang XD, Zhong XZ, et al. Preoperative ultrasound radiomics signatures for noninvasive evaluation of biological characteristics of intrahepatic cholangiocarcinoma. Acad Radiol 2020; 27: 785-797.

[23] Yao Z, Dong Y, Wu G, Zhang Q, Yang D, Yu JH, et al. Preoperative diagnosis and prediction of hepatocellular carcinoma: Radiomics analysis based on multi-modal ultrasound images. BMC Cancer 2018; 18: 1089.

[24] European Association for the Study of the Liver; European Association for the Study of the Liver. EASL Clinical Practice Guidelines: Management of hepatocellular carcinoma. J Hepatol 2018; 69: 182-236.

[25] Chan AWH, Zhong J, Berhane S, Toyoda H, Cucchetti A, Shi K, et al. Development of pre and post-operative models to predict early recurrence of hepatocellular carcinoma after surgical resection. $J$ Hepatol 2018; 69: 1284-1293.

[26] Rodríguez-Perálvarez M, Luong TV, Andreana L, Meyer T, Dhillon AP, Burroughs AK. A systematic review of microvascular invasion in hepatocellular carcinoma: diagnostic and prognostic variability. Ann Surg Oncol 2013; 20: 325-339.

[27] Sumie S, Kuromatsu R, Okuda K, Ando E, Takata A, Fukushima $\mathrm{N}$, et al. Microvascular invasion in patients with hepatocellular carcinoma and its predictable clinicopathological factors. Ann Surg Oncol 2008; 15: 1375-1382.

[28] Chen W, Zheng R, Zhang S, Zeng H, Xia C, Zuo T, et al. Cancer incidence and mortality in China, 2013. Cancer Lett 2017; 401: 63-71.

[29] Guo Y, Hu Y, Qiao M, Wang Y, Yu J, Li J, et al. Radiomics analysis on ultrasound for prediction of biologic behavior in breast invasive ductal carcinoma. Clin Breast Cancer 2018; 18: e335-e344.

[30] Lee SE, Han K, Kwak JY, Lee E, Kim EK. Radiomics of US texture features in differential diagnosis between triple-negative breast cancer and fibroadenoma. Sci Rep 2018; 8: 13546.

[31] Luo WQ, Huang QX, Huang XW, Hu HT, Zeng FQ, Wang W. Predicting breast cancer in breast imaging reporting and data system (BI-RADS) ultrasound category 4 or 5 lesions: A nomogram combining radiomics and BI-RADS. Sci Rep 2019; 9: 11921.

[32] Li Y, Liu Y, Zhang M, Zhang G, Wang Z, Luo J. Radiomics with attribute bagging for breast tumor classification using multimodal ultrasound images. J Ultrasound Med 2020; 39: 361-371.

[33] Yersal O, Barutca S. Biological subtypes of breast cancer: Prognostic and therapeutic implications. World J Clin Oncol 2014; 5: 412-424.

[34] Dent R, Trudeau M, Pritchard KI, Hanna WM, Kahn HK, Sawka CA, et al. Triple-negative breast cancer: clinical features and patterns of recurrence. Clin Cancer Res 2007; 13: 4429-4434.

[35] Cho N. Molecular subtypes and imaging phenotypes of breast cancer.
Ultrasonography 2016; 35: 281-288.

[36] Yu FH, Wang JX, Ye XH, Deng J, Hang J, Yang B. Ultrasoundbased radiomics nomogram: A potential biomarker to predict axillary lymph node metastasis in early-stage invasive breast cancer. Eur $J$ Radiol 2019; 119: 108658.

[37] Bray F, Ferlay J, Soerjomataram I, Siegel RL, Torre LA, Jemal A. Global cancer statistics 2018: GLOBOCAN estimates of incidence and mortality worldwide for 36 cancers in 185 countries. CA Cancer J Clin 2018; 68: 394-424.

[38] Chen LD, Li W, Xian MF, Zheng X, Lin Y, Liu BX, et al. Preoperative prediction of tumour deposits in rectal cancer by an artificial neural network-based US radiomics model. Eur Radiol 2020; 30: 1969-1979.

[39] Chen LD, Liang JY, Wu H, Wang Z, Li SR, Li W, et al. Multiparametric radiomics improve prediction of lymph node metastasis of rectal cancer compared with conventional radiomics. Life Sci 2018; 208: 55-63.

[40] Li X, Jiang F, Guo Y, Jin Z, Wang Y. Computer-aided diagnosis of gastrointestinal stromal tumors: a radiomics method on endoscopic ultrasound image. Int J Comput Assist Radiol Surg 2019; 14: 16351645.

[41] Greene FL. Tumor deposits in colorectal cancer: a moving target. Ann Surg 2012; 255: 214-215.

[42] Colombo PE, Patani N, Bibeau F, Assenat E, Bertrand MM, Senesse $\mathrm{P}$, et al. Clinical impact of lymph node status in rectal cancer. Surg Oncol 2011; 20: e227-233.

[43] Sashidharan P, Matele A, Matele U, Al Felahi N, Kassem KF. Gastrointestinal stromal tumors: a case report. Oman Med $J$ 2014; 29: $138-141$.

[44] Chen W, Zheng R, Baade PD, Zhang S, Zeng H, Bray F, et al. Cancer statistics in China, 2015. CA Cancer J Clin 2016; 66: 115-132.

[45] Liang J, Huang X, Hu H, Liu Y, Zhou Q, Cao Q, et al. Predicting malignancy in thyroid nodules: radiomics score versus 2017 American college of radiology thyroid imaging, reporting and data system. Thyroid 2018; 28: 1024-1033.

[46] Liu T, Zhou S, Yu J, Guo Y, Wang Y, Zhou J, et al. Prediction of lymph node metastasis in patients with papillary thyroid carcinoma: A radiomics method based on preoperative ultrasound images. Technol Cancer Res Treat 2019; 18: 1533033819831713.

[47] Liu T, Ge X, Yu J, Guo Y, Wang Y, Wang W, et al. Comparison of the application of B-mode and strain elastography ultrasound in the estimation of lymph node metastasis of papillary thyroid carcinoma based on a radiomics approach. Int J Comput Assist Radiol Surg 2018; 13: 1617-1627.

[48] Park VY, Han K, Lee E, Kim EK, Moon HJ, Yoon JH, et al. Association between radiomics signature and disease-free survival in conventional papillary thyroid carcinoma. Sci Rep 2019; 9: 4501.

[49] Tessler FN, Middleton WD, Grant EG, Hoang JK, Berland LL, Teefey SA, et al. ACR thyroid imaging, reporting and data system (TI-RADS): White paper of the ACR TI-RADS Committee. $J$ Am Coll Radiol 2017; 14: 587-595.

[50] Smith-Bindman R, Lebda P, Feldstein VA, Sellami D, Goldstein RB, Brasic N, et al. Risk of thyroid cancer based on thyroid ultrasound imaging characteristics: results of a population-based study. JAMA Intern Med 2013; 173: 1788-1796.

[51] Lim H, Devesa SS, Sosa JA, Check D, Kitahara CM. Trends in thyroid cancer incidence and mortality in the United States, 19742013. JAMA 2017; 317: 1338-1348.

[52] Liu Z, Zeng W, Liu C, Wang S, Xiong Y, Guo Y, et al. Diagnostic accuracy of ultrasonographic features for lymph node metastasis in papillary thyroid microcarcinoma: a single-center retrospective study. World J Surg Oncol 2017; 15: 32. 